This document is the accepted manuscript version of the following article:

Priebe, A., Xie, T., Pethö, L., \& Michler, J. (2020). Potential of gas-assisted time-of-flight

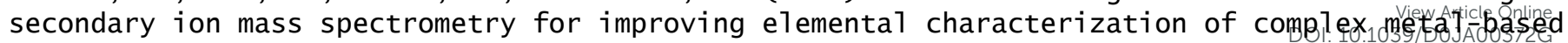
systems. Journal of Analytical Atomic Spectrometry. https://doi.org/10.1039/D0JA00372G

\title{
ARTICLE
}

\section{Potential of gas-assisted time-of-flight secondary ion mass spectrometry for improving elemental characterization of complex metal-based systems}

Received 00th January 20xx, Accepted 00th January 20xx

DOI: $10.1039 / \times 0 \times x 00000 x$

\author{
Agnieszka Priebe, ${ }^{* a}$ Tianle Xie, ${ }^{\mathrm{a}, \mathrm{b}}$ Laszlo Pethö $^{\mathrm{a}}$ and Johann Michler ${ }^{\mathrm{a}}$
}

\section{Introduction}

Maximizing the spatial resolution of Time-Of-Flight Secondary Ion Mass Spectrometry (TOF-SIMS) ${ }^{1,2}$ is currently one of the most important issues in the field of chemical analysis. In addition to improving the instrumental setup, i.e. adjusting the primary ion beam (a type of ions and beam parameters) with respect to the investigated specimen and optimizing the ion extraction optics, this can be achieved by developing techniques, which increase secondary ion generation, as the probability of element ionization determines the functionality of TOF-SIMS. The very high sensitivity in the order of ppm-ppb allows TOF-SIMS to detect even very small quantities of matter (i.e. corresponding peaks appear in mass spectra). However, a sufficiently high signal to noise ratio must be provided to characterize a sample's structure in 2D or 3D.

Until now, the highest TOF-SIMS lateral resolution ${ }^{5}$ below 20 $\mathrm{nm}$ was achieved using a BAM-L200 reference sample. Additionally, inorganic nanoparticles 7,8 were successfully analyzed in 3D. However, in both these cases, obtaining such

a. Empa, Swiss Federal Laboratories for Materials Science and Technology, Laboratory for Mechanics of Materials and Nanostructures, Feuerwerkerstrasse 39, CH-3602 Thun, Switzerland

*Corresponding author: agnieszka.priebe@empa.ch

b. College of Material Science and Engineering, Hunan University, 2 Lushan S Rd, Yuelu, Changsha, 410082, P.R. China

tElectronic Supplementary Information (ESI) available: See DOI: 10.1039/x0xx00000x impressive results was possible mainly due to the excellent ionization probability of aluminum and, most probably, would not be attainable in the case of weakly ionizing elements. This shows the necessity of combining TOF-SIMS with supporting methods, which can provide enhancement of secondary ion yields. As the ionization probability strongly depends on the chemical surface state (so called matrix effect ${ }^{9-11}$ ), it can be modified by introducing certain species to an experimental setup. The presence of oxygen ${ }^{2,9,12}$, water ${ }^{13-16}$ and fluorine ${ }^{16-19}$ have been shown to facilitate positive ion production, whilst $\mathrm{Cs}^{9,20-26}$ was found to increase negative ion yields.

The development of compact high-vacuum (HV) compatible TOF-SIMS detectors ${ }^{27-29}$, which can be integrated within Focused Ion Beam/Scanning Electron Microscope (FIB/SEM) instruments, gives a new opportunity for performing elemental analysis assisted using a supplementary gas delivered by a Gas Injection System (GIS). To verify the potential and limitations of a gas-assisted TOF-SIMS analysis, we have launched a systematic study on dedicated inorganic thin films in high vacuum conditions. Due to the complexity of such a multivariable analytical system, individual scientific problems were considered separately in subsequent stages and several dedicated thin film samples were synthesized for each problem. Stage 1 comprised of estimating the influence of water vapor and fluorine gas on ionization and sputtering processes of pure metals (i.e. $\mathrm{Cu}, \mathrm{Zr}, \mathrm{Ag}$ and $\mathrm{W})^{16}$. Cu showed the highest response to both gases, i.e. the ${ }^{63} \mathrm{Cu}^{+}$signal was enhanced by a factor of 10 and 510 for experiments conducted with water vapor and 
fluorine gas, respectively. Furthermore, $\mathrm{Zr}$ was found to provide a long and stable signal during gas-assisted FIB sputtering. This property made it an excellent candidate to serve as a base component of multi-element samples, which were analyzed in the next stages. The impact of fluorine gas on the spatial resolution of a $\mathrm{Cu} / \mathrm{Zr} / \mathrm{ZrCuAg}$ multilayer was addressed in Stage 2. At relatively high ${ }^{69} \mathrm{Ga}^{+}$ion beam energy lateral and depth resolution significantly increased during a single measurement. This is an important observation as, under standard vacuum conditions, two separate experiments must be performed, as the highest lateral resolution is achieved at $30 \mathrm{keV}$ and the highest depth resolution is obtained at low beam energies (usually $<5 \mathrm{keV}$ ). Moreover, it was observed that due to the different responses of $\mathrm{Zr}$ and $\mathrm{Ag}$ to the presence of fluorine gas as well as the fluorine-induced modification of metal-oxide formation, the mass interference between ${ }^{107} \mathrm{Ag}^{+}$and ${ }^{91} \mathrm{Zr}^{16} \mathrm{O}^{+}$ was separated ${ }^{19}$. This remarkable finding was observed for the first time and is expected to play an important role in the development of future TOF-SIMS protocols as currently mass interference remains one of the main drawbacks of this technique. In Stage 3 the matrix effect in Zr-containing twoelement alloys ( $\mathrm{ZrAl}, \mathrm{ZrSi}$ and $\mathrm{ZrCu}$ ) was investigated ( $\mathrm{Zr}$ was chosen based on the results obtained in Stage 1$)^{30}$, as a function of material components and boundary conditions (i.e. sensitivity to surface oxidation, contaminants and the proximity to the substrate). Over four times more $\mathrm{Zr}$ ions were generated in the Si matrix than in the case of the Cu matrix, and twice as many as in the case of the Al matrix. Moreover, the alloy components demonstrated different sensitivity to the thin film boundary conditions, resulting in different signal variations in depth profiles. These results served as the fundamentals for Stage 4 (presented in this publication) as they allowed the effects induced by water vapour or fluorine gas to be differentiated from the effects naturally resulting from the sample state (i.e. influence of $\mathrm{Al}, \mathrm{Si}$ and $\mathrm{Cu}$ on $\mathrm{Zr}$ ionization and vice versa, the influence of the substrate proximity, surface oxidation and surface contaminants on the ionization of the thin film components).

In this work, we show the potential and reliability of the novel gas-assisted TOF-SIMS method conducted under $\mathrm{HV}$ on $\mathrm{Zr}$ based alloy systems. The main concerns regarding the potential preferential sputtering of alloy components and modifications of measured isotope abundance resulting from introduced gases are verified. The obtained gas enhancement factors for TOF-SIMS signals and gas enhancement factors for sputtering rates of various alloys are compared to the results obtained in the case of pure elements. The sensitivity of element ionization to the thin film boundary conditions is discussed regarding the matrix type and applied gas. Our results prove that simultaneous gas co-injection during the TOF-SIMS analysis can be successfully conducted on thin films with thicknesses in the order of $100 \mathrm{~nm}$. The significantly increased secondary ion signals can help in improving spatial resolution. Moreover, it seems that supplementary gases can mitigate signal variations of uniform samples during depth profiling (i.e. inducing lower ionization sensitivity to the boundary conditions), making the
TOF-SIMS results more representative regarding Artięmple $_{\text {s. }}$ composition.

\section{DOI: 10.1039/D0JA00372G}

\section{Experimental}

\section{Materials}

Three two-element model samples were designed to verify the reliability of gas-assisted TOF-SIMS in the case of multicomponent systems. The term "multi-component" refers to the sample composition, as well as the presence of natural surface contaminants, such as hydrogen, carbon, oxygen and nitrogen, which can modify the sample's surface chemical state, and therefore affect the ionization process. Based on the experiments conducted on pure metallic samples ${ }^{16}, \mathrm{Zr}$ was chosen as the main invariant component for all considered alloys, as it has demonstrated the highest probability of generating secondary ions (up to three orders of magnitude higher when compared to the results of other measured elements) and the most stable signal distribution during depth profiling (i.e. the lowest sensitivity to the boundary conditions provides the most representative TOF-SIMS results regarding sample composition). To prevent mass interference (this concerns mass interference between isotopes of alloy components, their oxides, hydrides, hydroxides and fluorides), $\mathrm{Al}$, Si and Cu were used as alloying elements of Zr-based alloys. Moreover, Al has very low detection limits (around two orders of magnitude lower than $\mathrm{Cu}^{10}$ ), which was expected to provide detailed information on potential material segregation and associated gas-induced local signal variations. The combination of $\mathrm{Zr}$ and $\mathrm{Cu}$ in this analysis has an additional benefit, as it shows significant differences in sputtering rates. As was recently demonstrated, $\mathrm{Cu}$ sputters 2.7-3.0 times faster than $\mathrm{Zr}$ in the case of pure elements measured under standard vacuum conditions ${ }^{16}$. This trend changes when a supplementary gas is provided, i.e. $\mathrm{Zr}$ sputters 2.4 times and 1.4 times faster than $\mathrm{Cu}$ in the presence of water vapor and fluorine gas, respectively. Therefore, the $\mathrm{ZrCu}$ sample can serve for estimating if the entire alloy is sputtered uniformly or preferential sputtering (potentially leading to artefacts, especially during depth profiling) is induced by a supplementary gas.

The Zr-based alloys were fabricated via Physical Vapor Deposition (PVD) ${ }^{31}$. The detailed deposition parameters are described in our previous publication dedicated to studying the matrix effect in these samples ${ }^{30}$. A $3^{\prime \prime}$ sputter magnetron from MANTIS Deposition Ltd (Oxfordshire, United Kingdom) operated with $99.99 \%$ purity targets from Testbourne Ltd (Hampshire, England; Al, Si and $\mathrm{Zr}$ ) and EVOCHEM (Offenbach am Main, Germany; $\mathrm{Cu}$ ) was used to deposit around $100 \mathrm{~nm}$ thick thin films, with roughly 50 at.\% of $\mathrm{Zr}$ on $<100>$ single crystal Si wafers. In addition, $5 \mathrm{~nm}$ thick Au coatings were deposited on the samples' surfaces to prevent charging effects induced by FIB during TOF-SIMS measurements.

The exact thicknesses of the samples, $h_{\text {alloy, }}\left(h_{\text {zrAl }}=113 \mathrm{~nm}, h_{\text {zrsi }}\right.$ $=102 \mathrm{~nm}$ and $\mathrm{h}_{\mathrm{ZrCu}}=178 \mathrm{~nm}$ ) were obtained using SEM imaging on the samples' cross-sections. 


\section{Methods}

The gas-assisted TOF-SIMS measurements were performed using a HV-compatible Compact TOF (CTOF) detector from TOFWERK (Thun, Switzerland) which was mounted within a FIB/SEM dual beam system LYRA3 from Tescan (Brno, Czech Republic) equipped with a 5-line GIS. A continuous primary ion beam was used for sputtering and analysis (in contrast to the dedicated TOF-SIMS instruments, which usually operate with two separate pulsed beams). A $20 \mathrm{keV}$ energy mono-isotopic ${ }^{69} \mathrm{Ga}^{+}$ion beam at $(115 \pm 3) \mathrm{pA}$ ion current, $10 \mu \mathrm{s}$ dwell time and with the aperture of $100 \mu \mathrm{m}$ scanned over $10 \mu \mathrm{m} \times 10 \mu \mathrm{m}$ areas with $512 \times 512$ pixels and $2 \times 2$ binning. The elemental analysis of positive ions was performed only on the central $5 \mu \mathrm{m} \times 5 \mu \mathrm{m}$ Regions-Of-Interests (ROIs) to prevent potential artefacts originating from material re-deposition in the vicinity of the crater edges or from image drifts.

In the current commercially available instrumental setup, it is not possible to control the gas flux and the dose of injected gas cannot be directly measured. Assuming similar age of a gas precursor, the only variable, which can modify the amount of a given gas delivered to a sample surface, is the position of the GIS nozzle with respect to the sample surface. Therefore, to obtain comparable experimental data, all measurements were conducted at the same time (with the time difference up to several ours for a given gas and up to a day for experiments performed with different gases, i.e. water vapour or fluorine gas). This ensured the same or very similar experimental conditions such as the pressure in the vacuum chamber, the quality of gas precursors and the quality of $\mathrm{Ga}^{+}$beam source. The previously performed simulations ${ }^{16}$ with the GIS simulator ${ }^{32-34}$ have shown that the local water vapour pressure of $3 \times 10^{-3} \mathrm{mbar}$ and the local fluorine gas pressure of $6 \times 10^{-3}$ mbar at the FIB-TOF-SIMS ROIs. These values were obtained based on the operating gas pressure of $8.4 \times 10^{-6} \mathrm{mbar}$ in the case of water vapour and $1.0 \times 10^{-5} \mathrm{mbar}$ in the case of fluorine gas using the Knudsen number of 4.5. FIB-TOF-SIMS measurements are usually done at approx. $6-8 \times 10^{-6} \mathrm{mbar}$ background pressure of the vacuum chamber.

The data was acquired and processed using TOF-SIMS Explorer version 1.4.0.0 (TOFWERK, Thun, Switzerland). Mass calibration was performed using a primary ion beam signal peak $\left({ }^{69} \mathrm{Ga}^{+}\right)$and the most prominent isotopes of the samples, i.e. ${ }^{27} \mathrm{Al}^{+},{ }^{28} \mathrm{Si}^{+}$and ${ }^{90} \mathrm{Zr}^{+}$for $\mathrm{ZrAl},{ }^{28} \mathrm{Si}^{+}$and ${ }^{90} \mathrm{Zr}^{+}$for $\mathrm{ZrSi},{ }^{28} \mathrm{Si}^{+},{ }^{63} \mathrm{Cu}^{+}$and ${ }^{90} \mathrm{Zr}^{+}$for $\mathrm{ZrCu}$. In order to include peak tails in the analysis, for a given mass-to-charge ratio of an ion, $m / Q$, the secondary ion signals were collected from the $(1 \pm 0.5) \mathrm{m} / \mathrm{Q}$ ranges.

\section{Results and discussion}

The gas-assisted TOF-SIMS analysis in the case of samples consisting of more than one element is very complex due to the number of variables contributing to the ionization process. Considering a two-element alloy, first, there is a mutual effect of alloy components on their ionization, i.e. element 1 affects the ionization probability of element 2 and vice versa. Then, both elements can have different sensitivity to the alloy

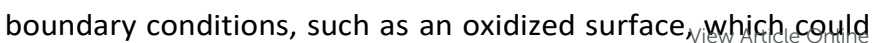
be potentially contaminated with elementS hateupan the atmosphere (mainly $\mathrm{H}, \mathrm{C}$ and $\mathrm{N}$ ) as well as the proximity to the sample substrate and the associated interface. Finally, the alloy components can show different response to the coinjected gas. To differentiate the effect of water vapor and fluorine gas on the ionization efficiency, first $\mathrm{ZrAl}, \mathrm{ZrSi}$ and $\mathrm{ZrCu}$ were analyzed under standard vacuum conditions. The main results of these studies ${ }^{30}$ show that: 1 ) the highest $\mathrm{Zr}$ ionization is observed in the presence of Si (over 4 times higher than in the case of the $\mathrm{Cu}$ matrix and over 2 times higher than in the case of the Al matrix), 2) ZrCu has the highest sputtering rate among the considered alloys (68\% higher than ZrAl and $53 \%$ higher than $\mathrm{ZrSi}$ ), 3) the ionization efficiency of alloy components varies across the thin films (i.e. in depth), suggesting different responses of the two elements to the aforementioned boundary conditions. In this work, we present how these features change due to the simultaneous injection of supplementary gases during the TOF-SIMS analysis. Moreover, the verification of the reliability of this method (especially in terms of gas-induced artefacts) for such multi-element systems is provided.

\section{Verification of sputtering process}

Sputtering yields depend on surface binding energy and energy transfer from the bombarding ion (in this case ${ }^{69} \mathrm{Ga}^{+}$) to the target atoms $\mathrm{s}^{35}$ (in this case $\mathrm{Al}, \mathrm{Si}, \mathrm{Cu}$ and $\mathrm{Zr}$ ). These two factors can be different for different atoms composing the analyzed multi-element target. Considering a two-element system of components $A$ and $B$, the ratio of their partial sputter yields (defined as the number of ejected atoms per incident ion) $Y_{A}$ and $Y_{B}$ is proportional to the ratio of their concentrations $N_{A}$ and $N_{B}$, respectively:

$$
\frac{Y_{A}}{Y_{B}}=r \frac{N_{A}}{N_{B}}
$$

The preferential sputtering of one element occurs when the sputtering factor $r \neq 1$, which is the case of the near-surface region (in the bulk $r$ converges to 1 ).

Additionally, the first results of gas-assisted TOF-SIMS measurements conducted on pure element thin films ${ }^{16}$ show that a gas enhancement factor for sputtering, $G A E_{S P}$, varies depending on target material and the type of gas. In the case of the metals $(\mathrm{Cu}, \mathrm{Zr}, \mathrm{Ag}$ and $\mathrm{W})$ considered in this study, the values of $G A E_{S P}$ were in the range of 0.9 to 6 , when the water vapor was injected in proximity to the sample's surface and in the range of 0.6 to 2.3 , when fluorine gas was used. This implies serious concerns about potential preferential sputtering in the case of multi-element systems. In order to verify this, the lateral elemental images of the samples' main isotopes (i.e. the pairs of ${ }^{27} \mathrm{Al}^{+}$and ${ }^{90} \mathrm{Zr}^{+},{ }^{28} \mathrm{Si}^{+}$and ${ }^{90} \mathrm{Zr}^{+}$or ${ }^{63} \mathrm{Cu}^{+}$and ${ }^{90} \mathrm{Zr}^{+}$) were studied at three different locations, i.e. 1) in a near-surface region, 2 ) in the middle of the thin film and 3 ) in the proximity to the Si substrate (Figure S1 in Supporting Information). The secondary ion signals were integrated over several frames corresponding to $10 \mathrm{~nm}$ thickness of the measured alloys. In general, the 
lateral elemental images obtained under standard vacuum conditions, in the presence of water vapor or fluorine gas do not show any statistically significant signal distributions (Figures S2S4). This indicates a lack of preferential sputtering in the case of $100 \mathrm{~nm}$ thick samples. Only in the case of $\mathrm{ZrCu}$, the ${ }^{63} \mathrm{Cu}^{+}$signal measured close to the interface between the $\mathrm{ZrCu}$ thin film and $\mathrm{Si}$ substrate is not homogeneous. However, this can be correlated with the total thickness of this thin film, which is much greater than the thickness of other samples (i.e. $h_{z r C u}=$ $178 \mathrm{~nm}$ while $h_{z \mathrm{rAl}}=113 \mathrm{~nm}$ and $h_{z r S i}=102 \mathrm{~nm}$ ).

\section{Influence of the co-injected gases on the depth profile distributions}

Regarding TOF-SIMS analysis of uniform samples, the importance of the matrix effect is particularly crucial in the case of depth profiles. Among potential surface contaminants, which are naturally present in the atmosphere, oxygen has the strongest effect on increasing the generation of positive secondary ions ${ }^{2,9,12}$. Furthermore, the proximity to the sample's substrate can modify the ionization process. These boundary condition can result in a local TOF-SIMS increase (or decrease, depending on a sample), which is not correlated with an increase (or decrease) in the amount of this material in the sample. Therefore, this can lead to data misinterpretation. As co-injecting a supplementary gas during a TOF-SIMS measurement adds additional variables to this already complicated multi-element system, it is important to verify to what extent the depth profile shapes are changed when compared to the results obtained under standard vacuum conditions. In our previous studies on pure elements ${ }^{16}$, it was observed that, in general, the magnitude of the signal was increased in the case of water vapor assisted TOF-SIMS, however the distribution shapes were similar (apart from the differences in sputtering rates) to those measured without any gas. In the case of fluorine-assisted measurements, the shape of the depth profiles significantly changed in the near-surface region. The signal peaks appearing in the first several frames, associated with surface oxidation ${ }^{16}$, disappeared. Additionally, the studies of matrix effect in two-element alloys (measured under standard vacuum conditions) ${ }^{30}$ show that different components of the alloys respond differently to the boundary conditions, meaning that the ratio of element 1 signal to element 2 signal varies across the sample.

The depth profiles of $\mathrm{ZrAl}, \mathrm{ZrSi}$ and $\mathrm{ZrCu}$ obtained during the gas-assisted TOF-SIMS measurements are given in Figure $\mathrm{S} 5$ and Figure S6. In most cases, the secondary ion signals of the main sample isotopes and the substrate were significantly higher when compared to the results obtained under standard vacuum conditions. To judge on the profile distributions and to estimate the location of the interface between the thin film and the substrate (i.e. given by the cross-point between the alloy signals and the substrate signal), the data was normalized to 1 (Figure 1). Regarding the TOF-SIMS measurements with water vapor, the ${ }^{27} \mathrm{Al}^{+}$signal of the $\mathrm{ZrAl}$ sample (Figure 1a) has two significant peaks on the thin film boundaries, the same as in the case when no gas was used. Here, however, the peak at the surface is higher by $41 \%$ than the one recorded close to the substrater (so

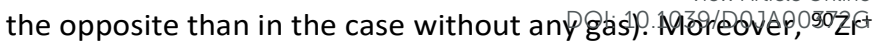
signal decreases steadily during the sputtering process. These two observations indicate at the possibility that some of the delivered water molecules accumulated at the bottom of the forming crater (i.e. the efficiency of the gas pumping process was lower there than on the flat sample surface located higher) decreasing the ionization process (as it is known that in case of some ionization-enhancing species there is a strict quantity criterion providing the maximum of sample signal increase $26,36,37$ ). The absence of the distinct peak in the proximity of the interface is observed in ${ }^{90} \mathrm{Zr}^{+}$signal for $\mathrm{ZrSi}$ (Figure 1c), as well as $\mathrm{ZrCu}$ (Figure 1e) samples. Additionally, in the latter cases, the data indicates that the presence of water vapor flattens the ${ }^{90} \mathrm{Zr}^{+}$signal distributions along the thin films (apart from the naturally oxidized upper-most sample surfaces, where the chemical composition is different than in the remaining part of the samples).

In the case of the fluorine gas-assisted experiments (Figure $1 \mathrm{~b}$, $d$ and $f$ ), the common tendency of all alloys, which is also consistent with the results of pure metal elements ${ }^{16}$, is the absence of the signal peak in the near-surface region. Instead, a significant signal deficiency is observed. The only exception to this rule occurs in the case of ${ }^{90} \mathrm{Zr}^{+}$signal for $\mathrm{ZrCu}$ alloy. It is worth noting that the surface peak of ${ }^{90} \mathrm{Zr}^{+}$signal is exactly in the location of the decreased ${ }^{63} \mathrm{Cu}^{+}$signal. Similarly to water vapor assisted measurements (Figure 1e), in the presence of fluorine gas the ${ }^{63} \mathrm{Cu}^{+}$signal increases in time, reaching the maximum value close to the interface with the Si substrate. Furthermore, considering that $\mathrm{ZrCu}$ is the thickest sample among all the considered samples, it might signify that the total amount of accumulated fluorine gas increased with the growing depth of the crater. This can potentially indicate that higher concentrations of fluorine lead to a higher ionization probability of $\mathrm{Cu}$ in the $\mathrm{Zr}$ matrix.

\section{Matrix effect in the complex multi-element system}

In our previous work ${ }^{30}$, the matrix effect in two-element alloys measured under standard vacuum conditions was presented. As it was already mentioned, although only two elements composed the single alloy, the analytical system was in fact a multi-element system. Element 1 affected ionization efficiency of element 2 and in return the ionization probability of element 1 depended on the presence of element 2. Additionally, elements such as $\mathrm{H}, \mathrm{C}, \mathrm{N}, \mathrm{O}$ (atmospheric gas components) and $\mathrm{Si}$ (substrate) can impact the secondary ion generation of the two elements. The situation becomes even more complex when a supplementary gas is injected. To verify the modifications of matrix effect in such systems, it is useful to represent the ratio of two of the most prominent alloy isotopes as a function of the sample thickness (Figure S7 and Figure S8). Regarding the ZrAl alloy (Figure $\mathrm{S} 7 \mathrm{a}$ and $\mathrm{b}$ ), the ${ }^{90} \mathrm{Zr}^{+} /{ }^{27} \mathrm{Al}{ }^{+}$ratio remains constant in the central part of the sample (i.e. between approx. $30 \mathrm{~nm}$ and $90 \mathrm{~nm}$ ) under all considered conditions, however, its value changes depending on whether the sample was measured without (i.e. around 1.2) or with gas (around 0.9 and 0.5 in the 


\section{Measurements with water vapour}

a) $\mathrm{ZrAl}-27 \mathrm{Al}+$ (water, avg) $-28 \mathrm{Si}+$ (water, avg) $-90 \mathrm{Zr}+$ (water, avg)

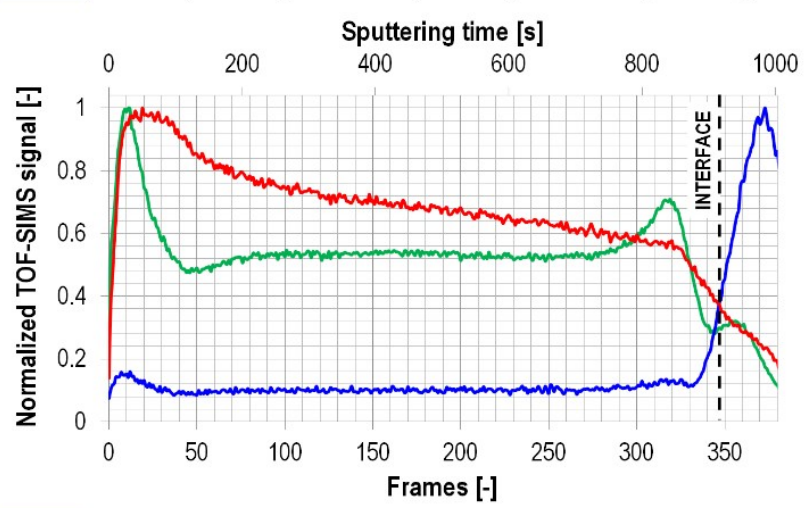

c) $\mathrm{ZrSi}$
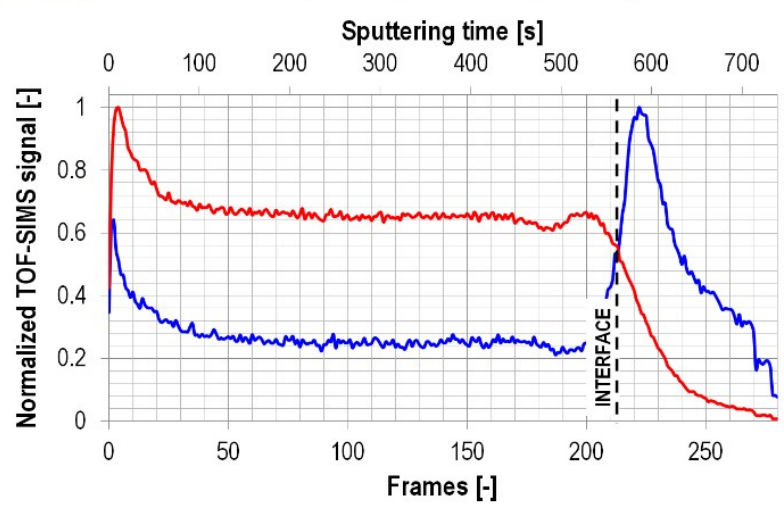

e) $\mathrm{ZrCu}-28 \mathrm{Si}+$ (water, avg) $-63 \mathrm{Cu}+$ (water, avg) $-90 \mathrm{Zr}+$ (water, avg)

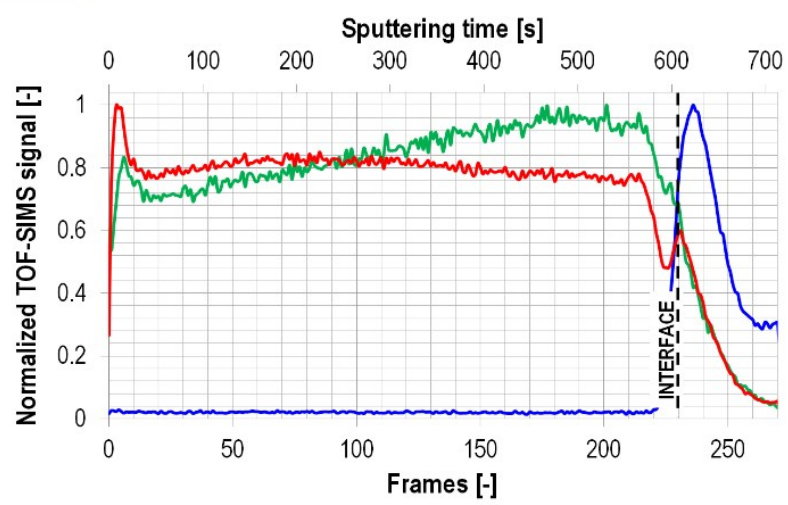

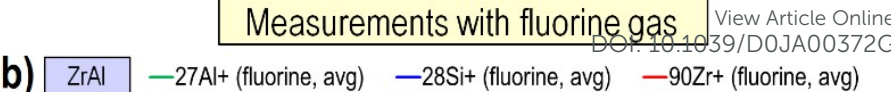

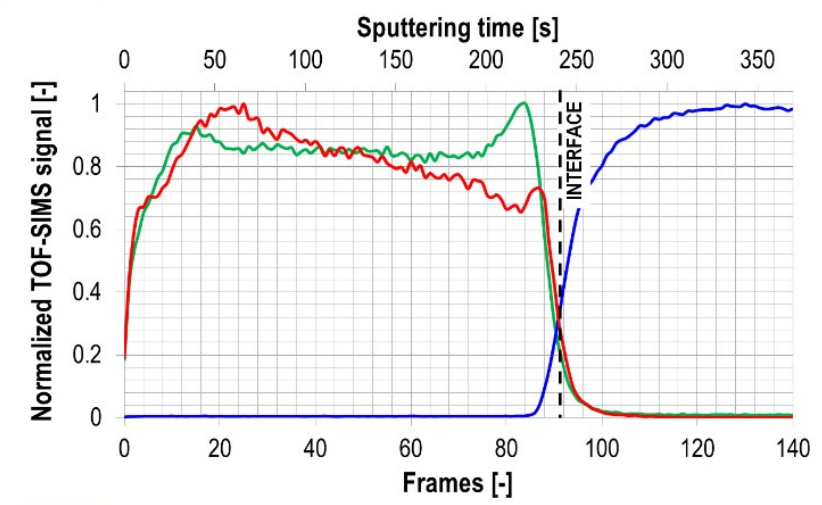

d) $\mathrm{ZrSi} \quad-28 \mathrm{Si}+$ (fluorine, avg) $-90 \mathrm{Zr}+$ (fluorine, avg)

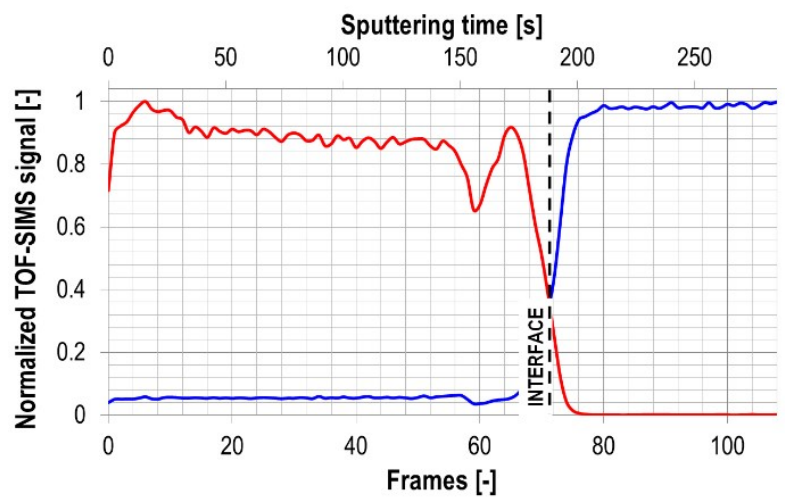

f) $\mathrm{ZrCu}-28 \mathrm{Si}+$ (fluorine, avg) $-63 \mathrm{Cu}+$ (fluorine, avg) $-90 \mathrm{Zr}+$ (fluorine, avg)

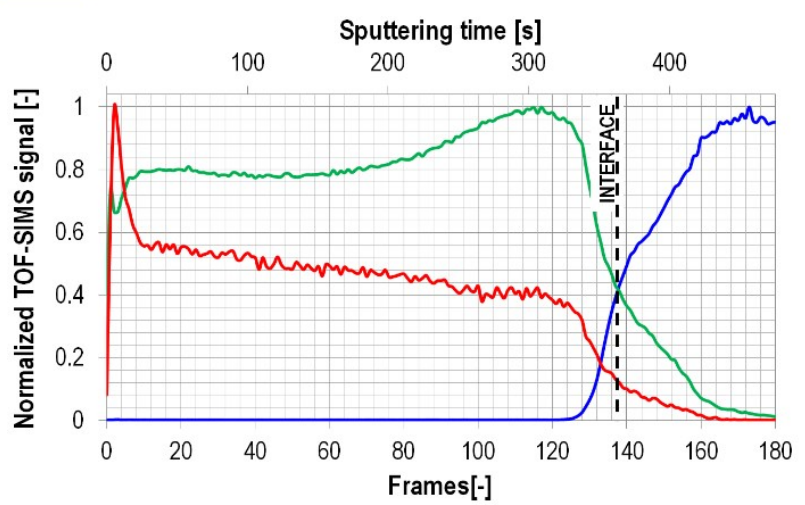

Figure 1. Normalized to 1 depth profiles of the most prominent isotopes of $\mathrm{ZrAl}$ (a and b), ZrSi (c and d) and ZrCu (e and f) alloys acquired during water vapor- and fluorine gasassisted TOF-SIMS measurements. The black dashed line indicates the estimated location of the interface between the alloy thin film and the Si substrate.

case of water vapor and fluorine gas, respectively). The data suggest that the effect of fluorine gas on the ZrAl ionization process is dominant with respect to the surface contaminants, as the signal ratio variations in the first $30 \mathrm{~nm}$ of the thin film are the smallest (Figure $\mathrm{S} 8 \mathrm{~b}$ ). In contrast, higher signal variations on the thin film boundaries were observed when water vapor was used (Figure S8a). The ${ }^{90} \mathrm{Zr}^{+} /{ }^{28} \mathrm{Si}^{+}$ratio equals around 30-35 in the central part of $\mathrm{ZrCu}$ thin film under standard vacuum conditions and decreases to around 9 when water vapor was used and to around 4 when fluorine was injected. Regardless on the type of gas, the differences in ionization sensitivity of $\mathrm{Zr}$ and $\mathrm{Si}$ significantly diminish at the thin film boundaries. In the case of water vapor, (Figure S8c) only around $9 \mathrm{~nm}$ of external parts of the alloy are affected (compared to $20 \mathrm{~nm}$ of surface region and $10 \mathrm{~nm}$ of the nearsubstrate region, which have lower signal in the case when no gas was used). With respect to fluorine gas (Figure S8d), only the deepest $20 \mathrm{~nm}$ of $\mathrm{ZrSi}$ alloy are changed when compared to the remaining part of the sample, i.e. no significant change in the ${ }^{90} \mathrm{Zr}^{+} / 28 \mathrm{Si}^{+}$ratio is observed on the sample's surface. The ${ }^{90} \mathrm{Zr}^{+} / 63 \mathrm{Cu}^{+}$ratio of the reference measurement without any gas (Figure S8e) is around 17 in the central part of the sample. High signal variations are observed across the thin film with a signal drop within around $10-25 \mathrm{~nm}$ thick regions of the sample 
boundaries. Regarding both gas-assisted measurements, the ${ }^{90} \mathrm{Zr}^{+} /{ }^{63} \mathrm{Cu}^{+}$ratios are lower when compared to the results obtained under standard vacuum conditions, i.e. 5-7 times in the case of water vapor and 213-340 times in the case of fluorine gas. These ranges, varying by up to $60 \%$, result from two signal plateau, which can be distinguished in the signal distributions. The ${ }^{90} \mathrm{Zr}^{+} /{ }^{63} \mathrm{Cu}^{+}$ratio signal increase was recorded in the uppermost surface of the sample surface and can be correlated with the oxidized layer. In contrast to the waterassisted measurement, no sudden signal decrease was measured in the vicinity of the Si substrate during the fluorineassisted experiment.

In conclusion, the conducted analysis indicates that the depth profiles acquired during fluorine-gas assisted TOF-SIMS measurements are more representative with respect to the thin film composition than the depth profiles obtained under standard vacuum conditions and in the presence of water vapor. It seems that, in general, there are fewer signal variations at the sample boundaries with respect to the signals measured in the central parts of the alloys. Moreover, the data suggests that $\mathrm{Zr}$ ionization efficiency in 2-element alloys is less sensitive to the presence of $\mathrm{Si}$ originating from the substrate when supplementary gas is provided.

\section{Gas enhancement factors for TOF-SIMS signals and sputtering rates}

The gas enhancement factor TOF-SIMS signal, GAE is defined as a ratio of total integrated isotope signal measured across a thin film (i.e. until the time of reaching an interface with the substrate, $\left.t_{\text {int,gas }}\right)$ during a gas-assisted TOF-SIMS measurement, $S_{x \mid+, \text { gas }}$ (where ${ }^{x l^{+}}$represents an isotope of an element), to the corresponding total signal acquired under standard vacuum conditions, $S_{x \mid+, \text { ref }}$.

$$
G A E_{T O F-S I M S, x I+, g a s}=\frac{\int_{t=0}^{t=t_{\text {int }}} S_{x I+, g a s}(t) d t}{\int_{t=0}^{t=t_{\text {int }}} S_{x I+, r e f}(t) d t} .
$$

The total TOF-SIMS signal is proportional to the useful ion yield, $Y_{\text {useful, defined as a number of detected secondary ions per }}$ number of all sputtered atoms of a given element. As, the corresponding useful yields were acquired from the same volumes (i.e. $5 \mu \mathrm{m} \times 5 \mu \mathrm{m} \times h_{\text {alloy }}$ ), the value of the gas enhancement factor can also be expressed as:

$$
G A E_{\text {TOF-SIMS, xI }, \text { gas }}=\frac{Y_{\text {useful,gas }}}{Y_{\text {useful,ref }}} .
$$

In general, the use of absolute values of useful ion yields is not practical due to their dependency on matrix effect and detector parameters ${ }^{38-40}$. Therefore, in order to assess the gas influence on the ionization efficiency, it seems much more reasonable to operate with the relative values.

The values of gas enhancement factors for TOF-SIMS signals in the case of water vapor, GAE TOF-SIMS, $x 1+, w v$, and fluorine gas, GAE $E_{T O F-S I M S, x l+f,}$ are given in Table 1 and Table 2, respectively (the values of $t_{\text {int, ref, }} t_{\text {int,gas }}, S_{x \mid+, \text { ref }}$ and $S_{x \mid+, \text { gas }}$ used for calculations can be found in Table S1 and Table S2). In most cases (apart from the $\mathrm{ZrCu}$ alloy exposed to fluorine gas), the $\mathrm{Zr}$ ionization efficiency in a metallic/metalloid matrix is enhapced , when $_{\text {in }}$ a supplementary gas is provided. ThisOlis 0.10 pa/interesting observation showing the importance of the matrix, as in case of pure $\mathrm{Zr}$ the co-injection of water vapor resulted in $82 \%$ lower generation of ${ }^{90} \mathrm{Zr}^{+}$ions ${ }^{16}$ and fluorine gas induced a $27 \%$ drop. The highest gas enhancement factor for ${ }^{90} \mathrm{Zr}^{+}$signal $(5.34 \pm 0.05)$ was obtained in the case of the $\mathrm{ZrSi}$ alloy measured in the fluorine gas atmosphere. Among the considered matrix elements, $\mathrm{Cu}$ barely modifies $\mathrm{Zr}$ ionization in the case of fluorine gas-assisted measurements $\left(0.81 ; G A E_{\text {TOF-SIMS, } 90 Z r+, f}=0.73\right.$ in the case of pure metal).

As was previously mentioned, during gas-assisted elemental characterization of complex samples, there is a cumulative matrix effect, i.e. the effect of supplementary gas superimposes on the effect of the sample matrix. Under standard vacuum conditions, the most beneficial combination of elements leading to the highest generation of ${ }^{90} \mathrm{Zr}^{+}$ions was observed for $\mathrm{ZrSi}$ (over four times higher than in the case of the $\mathrm{ZrCu}$ alloy and over two times higher when compared to the ZrAl alloy ${ }^{30}$ ). This tendency changes when gas is provided. The $\mathrm{Zr}$ ionization efficiency is $23 \%$ higher in the Al matrix than in the Si matrix and 2.4 times higher than in the $\mathrm{Cu}$ matrix regarding the measurements conducted in a water vapor atmosphere (Table $\mathrm{S} 1)$. Interestingly, in the case of fluorine gas-assisted analysis, the same number of ${ }^{90} \mathrm{Zr}^{+}$ions is produced in the Al matrix as in the Si matrix. These values are higher by a factor of 2.7 when compared to the Cu matrix (Table S2).

In addition to the gas enhancement factor for TOF-SIMS signal, another useful parameter for comparing the influence of gas during the FIB-TOF-SIMS analysis is the gas enhancement factor for sputtering rates, $G A E_{S P, \text { gas, }}$ given by:

$$
G A E_{S P, g a s}=\frac{Y_{S P, g a s}}{Y_{S P, \text { ref }}} .
$$

$Y_{S P}$ denotes the sputter rate defined as the number of sputtered atoms from the target per unit of time ${ }^{41}$. As the sputtered volumes in the case of individual thin films measured without and with gas are the same, so are the number of sputtered atoms. Therefore, Equation 4 can be simplified to:

$$
G A E_{S P, g a s}=\frac{t_{\text {int }, \text { ref }}}{t_{\text {int, gas }}} .
$$

Regarding the $\mathrm{Zr}$-based two-element alloys considered in this work, the co-injecting of water vapor resulted in decreasing the efficiency of the FIB sputtering process with ${ }^{69} \mathrm{Ga}^{+}$primary ion beam by $25 \%-46 \%$. This is an interesting observation as the experiments conducted on pure $\mathrm{Zr}$ metal ${ }^{16}$ has shown that coinjection of water vapour has increased the $\mathrm{Zr}$ sputtering efficiency by a factor of $6 \pm 2$. Since both series of measurements were performed at the same primary ion beam energy and current, the decreased sputter rates of $\mathrm{Zr}$-based alloys most likely result from the presence of alloying elements (i.e. Al, Si and $\mathrm{Cu}$ ), their individual response to water vapour and/or the structural configuration of the alloys. The most significant drop in sputtering rate was observed in the case of ZrAl $\left(G A E_{S P, w v, Z r A l}=0.54 \pm 0.03\right)$. ZrSi was the least sensitive to the 
influence of gas with $G A E_{S P, w v, Z r s i}=0.75 \pm 0.04$. This trend is opposite to the results obtained for pure $\mathrm{Zr}$, sputtering rate of which increased approx. six fold. In contrast to water vapor, fluorine gas increased the sputtering rates almost by a factor of 2 (77\%-90\%, GAE $E_{S P, f, Z r A l}=1.9 \pm 0.1, G A E_{S P, f, Z r S i}=1.77 \pm 0.08$ and $\left.G A E_{S P, f, Z r C U}=1.89 \pm 0.08\right)$. These results are similar to the value obtained for pure $\mathrm{Zr}$, i.e. $G A E_{S P, f, Z r}=2.3 \pm 0.2^{16}$.

\section{Isotope abundance in gas-assisted elemental analysis}

The simultaneous introduction of gas molecules in proximity to a sample's surface during ion bombardment carries a risk of generating new ions that could potentially mass-interfere with the sample's isotopes, and therefore, risk the formation of artefacts. To determine the presence of these phenomena, the

Table 1. Summary of TOF-SIMS signal ratios, gas enhancement factors for TOF-SIMS signals, GAE TOF-SIMS analysis $(w v)$.

\begin{tabular}{|c|c|c|c|c|c|}
\hline \multirow{2}{*}{ Alloy } & \multicolumn{2}{|c|}{ TOF-SIMS signal ratios [-] } & \multicolumn{2}{|c|}{ Gas enhancement factor for TOF-SIMS signal [-] } & $\begin{array}{c}\text { Gas enhancement factor for sputtering rates } \\
{[-]}\end{array}$ \\
\cline { 2 - 6 } & $\frac{S_{90 Z r+, r e f}}{S_{x M+, r e f}}$ & $\frac{S_{90 Z r+, w v}}{S_{x M+w v}}$ & $G A E_{T O F-S I M S, 90 Z r+w v}$ & $G A E_{T O F-S I M S, x M+, w v}$ & $G A E_{S P, w v}$ \\
\hline $\mathrm{ZrAl}$ & $1.00 \pm 0.08$ & $0.84 \pm 0.01$ & $2.21 \pm 0.06$ & $2.7 \pm 0.2$ & $0.54 \pm 0.03$ \\
\hline $\mathrm{ZrSi}$ & $22 \pm 4$ & $8.5 \pm 0.9$ & $1.29 \pm 0.07$ & $3.4 \pm 0.7$ & $0.75 \pm 0.04$ \\
\hline $\mathrm{ZrCu}$ & $14.1 \pm 0.9$ & $2.85 \pm 0.05$ & $3.1 \pm 0.2$ & $15.1 \pm 0.3$ & $0.69 \pm 0.02$ \\
\hline
\end{tabular}

The total TOF-SIMS signal measured across the thin film is given with S. M denotes Al, Si or Cu for ZrAl, ZrSi and ZrCu, respectively, and $x$ stands for 27,28 and 63 , correspondingly. The values measured without any gas are marked with ref index. The data used for calculations is given in Table S1 in the Supporting Information.

Table 2. Summary of TOF-SIMS signal ratios, gas enhancement factors for TOF-SIMS signals, GAE TOF-SIMS,f, and for sputtering rates, GAESP,f, obtained during fluorine-gas assisted TOFSIMS analysis (f).

\begin{tabular}{|c|c|c|c|c|c|}
\hline \multirow{2}{*}{ Alloy } & \multicolumn{2}{|c|}{ TOF-SIMS signal ratios [-] } & \multicolumn{2}{|c|}{ Gas enhancement factor for TOF-SIMS signal [-] } & Gas enhancement factor for sputtering rates [-] \\
\cline { 2 - 6 } & $\frac{S_{90 Z r+, r e f}}{S_{x M+, \text { ref }}}$ & $\frac{S_{90 Z r+, f}}{S_{x M+, f}}$ & $G A E_{T O F-S I M S, 90 Z r+f}$ & $G A E_{T O F-S I M S, x M+, f}$ & $G A E_{S P, f}$ \\
\hline $\mathrm{ZrAl}$ & $1.1 \pm 0.2^{*}$ & $0.49 \pm 0.01$ & $1.18 \pm 0.06$ & $2.6 \pm 0.3$ & $1.9 \pm 0.1$ \\
\hline $\mathrm{ZrSi}$ & $24 \pm 2^{*}$ & $3.4 \pm 0.6$ & $5.34 \pm 0.05$ & $3.8 \pm 0.8$ & $1.77 \pm 0.08$ \\
\hline $\mathrm{ZrCu}$ & $15.1 \pm 0.7 *$ & $0.694 \pm 0.004$ & $0.81 \pm 0.05$ & $176 \pm 15$ & $1.89 \pm 0.08$ \\
\hline
\end{tabular}

The data used for calculations is given in Table S2 in the Supporting Information.

occurred. Additionally, the ${ }^{96} \mathrm{Zr}^{+}$signal can mass overlap with the ${ }^{69} \mathrm{Ga}^{27} \mathrm{Al}^{+}$ion signal. A similar situation occurs for $\mathrm{ZrSi}$, where the deviation in measured $\mathrm{Zr}$ isotope abundance can result from the mass interference between ${ }^{91} \mathrm{Zr}^{+}$and ${ }^{28} \mathrm{Si}_{2}{ }^{16} \mathrm{O}_{2}{ }^{1} \mathrm{H}_{3}{ }^{+}$, as well as the mass interference between ${ }^{92} \mathrm{Zr}^{+}$and ${ }^{28} \mathrm{Si}^{16} \mathrm{O}_{4}{ }^{+}$. Slightly larger differences between $\mathrm{Zr}$ isotope abundance measured under standard vacuum and water vapor conditions are observed in the case of the $\mathrm{ZrCu}$ alloy. The ${ }^{91} \mathrm{Zr}^{+}$signal can massinterfere with the ${ }^{63} \mathrm{Cu}^{16} \mathrm{O}^{12} \mathrm{C}^{+}$signal and the signal at mass 94 can originate from ${ }^{94} \mathrm{Zr}^{+}$ions as well as from ${ }^{65} \mathrm{Cu}^{16} \mathrm{O}^{12} \mathrm{C}^{1} \mathrm{H}^{+}$ions. The moment of reaching the interface between the thin film and the substrate can be well-estimated based on the depth profiles. However, the signal distributions do not stop or appear abruptly but are spread across the sputtering time. Therefore, Si substrate ions can also potentially contribute to mass interference. This can explain the modified measured
$\mathrm{Zr}$ (the heaviest and the only common element ticlefnall

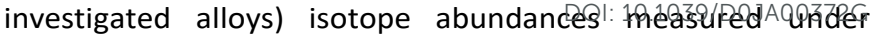
standard vacuum conditions, in the presence of water vapor and fluorine gas were compared to the natural $\mathrm{Zr}$ isotope abundance (Figure S9). The varying alloy components (i.e. Al, Si and $\mathrm{Cu}$ ) were chosen to prevent mass interference with $\mathrm{Zr}$. vapor are comparable, indicating that the observed deviation from the natural isotope abundance can be correlated with the presence of oxygen. Considering that in both cases, the ${ }^{91} \mathrm{Zr}^{+}$ion contribution is elevated by almost $4 \%$, this leads to the conclusion that the mass interference with ${ }^{27} \mathrm{Al}^{16} \mathrm{O}_{4}{ }^{+}$ions contribution of ${ }^{91} \mathrm{Zr}^{+}$ions having the same mass as ${ }^{63} \mathrm{Cu}^{28} \mathrm{Si}^{+}$. ${ }^{92} \mathrm{Zr}^{+}$and ${ }^{63} \mathrm{Cu}^{29} \mathrm{Si}^{+}$also have the same masses, however ${ }^{29} \mathrm{Si}$ isotope constitutes only a minor fraction of the total $\mathrm{Si}$ ions ( $4.7 \%$ compared to $92.2 \%$ of ${ }^{28} \mathrm{Si}$ ). Although a cluster of five water ions $\left({ }^{16} \mathrm{O}_{5}{ }^{1} \mathrm{H}_{10}{ }^{+}\right)$has the same mass as ${ }^{90} \mathrm{Zr}^{+}$, the mass interference seems to be highly unlikely, as in the case of measurements without any gas, and with water vapor the measured ${ }^{90} \mathrm{Zr}^{+}$contribution was lower than expected.

The most remarkable outcome of comparing isotope abundance was observed in the case of fluorine gas-assisted TOF-SIMS experiments. The maximum divergence from the natural isotope abundance of the most prominent ${ }^{90} \mathrm{Zr}^{+}$isotope reaches only $0.2 \%$ (whilst the minimum error of estimating total $S_{90 z r+}$, which originates in the uncertainty of the interface location, is $0.65 \%$, see Table S1 and Table S2) and maximum $0.7 \%$ in the case of minor isotopes. As we have postulated 
before ${ }^{19}$, this can be correlated with the higher electron affinity of fluorine $(322 \mathrm{~kJ} / \mathrm{mol})$ when compared to oxygen electron affinity $(141 \mathrm{~kJ} / \mathrm{mol})^{42}$, which can result in preferable formation of $\mathrm{Zr}-\mathrm{F}$ ions and lower probability of generating oxygencontaining ions. Consequently, this can lead to the separation of mass interference.

Summary of gas-assisted TOF-SIMS measurements conducted on pure metals and Zr-based alloys

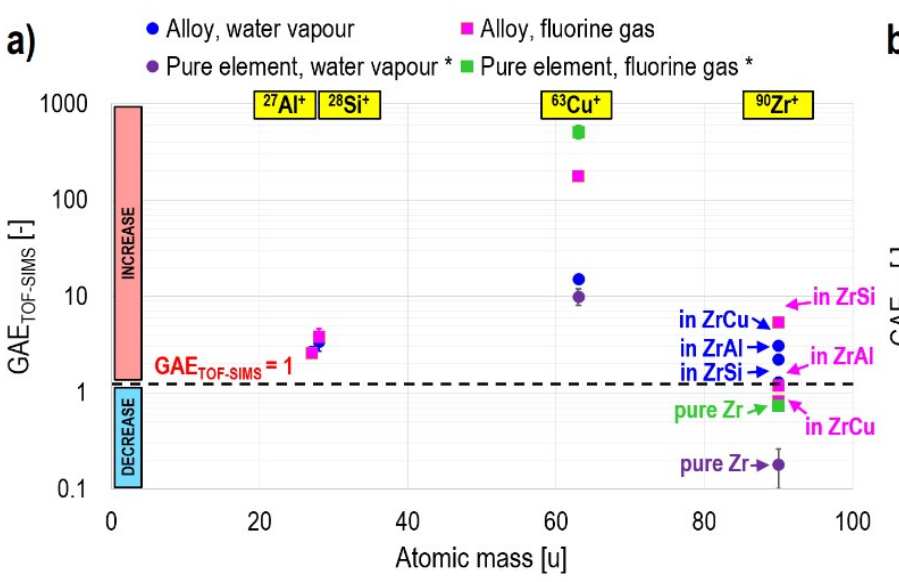

provided and the sequence of the ratio magnitudes nlins

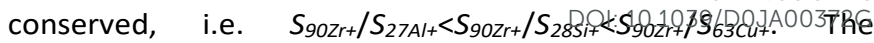
difference between the signal ratios is the smallest in the case of fluorine gas-assisted TOF-SIMS analysis.

\section{Conclusions}

The comprehensive studies conducted on inorganic twoelement alloys prove the potential of the GIS-upgraded TOFb) - Alloy, water vapour $\quad$ - Pure element, water vapour * $\quad$ = Pure element, fluorine gas *

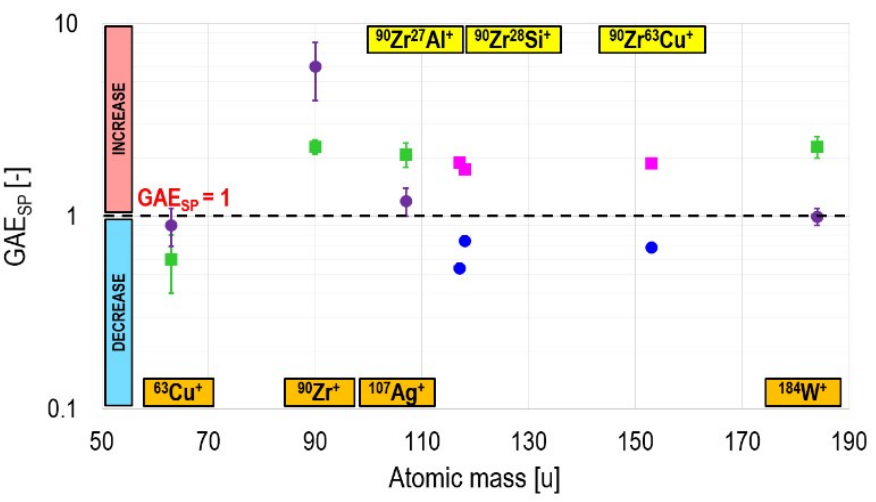

Figure 2. Summary of gas enhancement factors for TOF-SIMS signal (a) and gas enhancement factors for sputtering (b) in the case of pure metals (source: Priebe et al. ${ }^{16}$ ) and Zrbased two-element alloys. The dashed line denotes $G A E=1$ meaning no gas-induced changes.

Despite decades of studies, several theoretical models and substantial quantities of experimental data, the ionization process and matrix effect are still not completely understood ${ }^{11,43-45}$. The absence of a uniform ionization model capable of explaining the generation of secondary ions implies the necessity of performing systematic empirical studies on the materials of interest. As demonstrated in our previous work $^{16,19,30}$, the ionization efficiency and response to the provided gas is element dependent. Moreover, in the case of complex materials, the sample components can completely invert the influence of co-injected gas (e.g., from secondary ion signal enhancement to its decrease). Additionally, the quantity of gas delivered to the sample's surface can modify the magnitude of generated secondary ions and affect depth profile shapes.

The summary of GAE TOF-SIMS and $G A E_{S P}$ values of all gas-assisted TOF-SIMS measurements conducted within this systematic study (i.e. on single-element pure metallic thin films ${ }^{16}$ and twoelement $\mathrm{Zr}$-based alloys) is provided in Figure 2. For a given element, the values of GAETOF-SIMS can vary by several orders of magnitude depending on the type of gas used and sample chemical state (pure element or complex compound element component). Regarding the $G A E_{S P}$ values, the same gas can induce either an increase or decrease in material removal during FIB milling, depending on the material type. However, in most cases, fluorine gas enhanced the sputter process.

A comparison of $S_{902 r+} / S_{X M+}$ signal ratios measured under standard vacuum conditions and during gas-assisted measurements is shown in Figure S10. The trend in response to the experimental conditions can be observed, i.e. in all cases the signal ratio decreases when the supplementary gas was
SIMS for improving elemental characterization of complex systems. This novel experimental approach allows to significantly enhance secondary ion generation, which provides improved statistical data and higher quality elemental imaging. In the case of $100 \mathrm{~nm}$ thick thin films, no secondary ion distribution in the lateral plane (verified on different sample depths) was observed. This allows to exclude the possibility of preferential material sputtering during the gas-assisted TOFSIMS measurement, which was one of the main concerns regarding the presented technique. The increase in count rates enables obtaining depth profiles with reduced statistical signal fluctuations. Consequently, this improves the depth resolution and, in general, allows assessing the location of interfaces more precisely. Additionally, fluorine gas seems to modify the metaloxygen interactions, due to its higher electron affinity. This results in lower element signal variations in the proximity of the sample's edges leading to more reliable sample composition representation during depth profiling (e.g. the signal peak commonly occurring at the beginning of FIB sputtering does not result from higher element quantity, but from sample surface oxidation, and usually disappears once fluorine gas has been provided). In general, water vapor and fluorine gas can help in mitigating variations in $S_{90 z r+} / S_{X M+}$ ratios close to the thin film surface and substrate interface in the case of homogenous alloys. The introduction of additional elements (in this case, the gas precursor components) to an experimental system always carries the risk of implementing new ions, which could potentially mass-interfere with the sample's isotopes. However, the comparison between isotope abundances obtained during water vapor-assisted measurements and under standard vacuum conditions is similar (and biased with $3 \%$ deviation 
from the natural isotope abundance in the case of $\mathrm{Zr}$ ). Remarkably, the provided fluorine gas significantly improves the accuracy of measured $\mathrm{Zr}$ isotope abundance, which in the case of the most prominent isotope ${ }^{90} \mathrm{Zr}^{+}$is biased with only $0.2 \%$ error.

The calculated gas enhancement factors for TOF-SIMS signals and sputtering rates show the importance of the element type, alloy matrix and type of supplementary gas on the ionization process and sputtering efficiency. Both these values are characteristic and element dependent.

In conclusion, the combination of TOF-SIMS and GIS is a significant step toward maximizing secondary ion signals. Gas type optimization, its quantity and primary ion beam parameter selection as a function of studied material is expected to play an important role in reaching new resolution records for elemental characterization techniques. However, it should be noted that additional effects/artefacts may appear due to the complex matrix effect in multi-element systems. Therefore, it would be prudent to always conduct a reference measurement under standard vacuum conditions for comparison. The conclusions section should come in this section at the end of the article, before the acknowledgements.

\section{Conflicts of interest}

There are no conflicts to declare.

\section{Acknowledgements}

The EMPAPOSTDOCS-II program received funding from the European Union's Horizon 2020 research and innovation programme under the Marie Skłodowska-Curie grant agreement number 754364 . The TOF-SIMS measurements were performed in the frame of Swiss Commission for Technology and Innovation (CTI) project (no.: 25592.1 PFNM-NM). The authors would like to express their gratitude to TOFWERK (Thun, Switzerland) for providing access to the TOF-SIMS instrumentation.

\section{References}

1 A. Benninghoven, F. G. Rudenauer and H. W. Werner, Surf. Interface Anal., 1987, 10, 435.

2 J. C. Vickermann and D. Briggs, ToF-SIMS: Materials Analysis by Mass Spectrometry, IM Publications LLP, West Sussex, United Kingdom, 2nd edn., 2013.

3 S. Fearn, An introduction to time-of-flight secondary ion mass spectrometry (ToF-SIMS) and its application to materials science, Morgan \& Claypool Publishers, San Rafael, CA, USA, 2015.

4 J. Kuo, Electron Microscopy: Methods and Protocols (Methods in Molecular Biology), Humana Press, Totowa, New Jersey, 2nd edn., 2007.

5 F. Kollmer, W. Paul, M. Krehl and E. Niehuis, Surf. Interface Anal., 2013, 45, 312-314.

6 M. Senoner, A. Maaßdorf, H. Rooch, W. Österle, M.
Malcher, M. Schmidt, F. Kollmer, D. Paul, V. D Hodoreabane

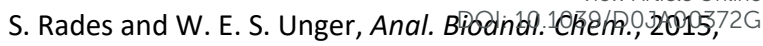
407, 3211-3217.
7

9

A. Priebe, J.-P. Barnes, T. E. J. Edwards, L. Pethö, I. Balogh and J. Michler, Anal. Chem., 2019, 91, 11834-11839 Article.

A. Priebe, Barnes Jean-Paul, T. E. J. Edwards, E. Huszár, L. Pethö and J. Michler, Anal. Chem., 2020, 92, 12518-12527. P. Van de Heide, Secondary Ion Mass Spectrometry: An Introduction to Principles and Practices, 2014. A. Benninghoven, ChemInform, 1994, 1023.

V. R. Deline, W. Katz, C. A. Evans and P. Williams, Appl. Phys. Lett., 1978, 33, 832-835.

A. Priebe, G. Audoit and J. P. Barnes, Ultramicroscopy, 2017, 173, 10-13.

S. Sheraz, A. Barber, J. S. Fletcher, N. P. Lockyer and J. C. Vickerman, Anal Chem, 2013, 2-3.

S. Sheraz, A. Barber, I. B. Razo, J. S. Fletcher, N. P. Lockyer and J. C. Vickerman, Surf. Interface Anal., 2014, 46, 51-53. S. Sheraz Née Rabbani, I. B. Razo, T. Kohn, N. P. Lockyer and J. C. Vickerman, Anal. Chem., 2015, 87, 2367-2374. A. Priebe, I. Utke, L. Pethö and J. Michler, Anal. Chem., 2019, 91, 11712-11722.

A. Wucher, Appl. Surf. Sci., 2006, 252, 6482-6489.

S. Ghalab and A. Wucher, Nucl. Instruments Methods Phys. Res. Sect. B Beam Interact. with Mater. Atoms, 2004, 226, 264-273.

A. Priebe, L. Pethö and J. Michler, Anal. Chem., 2020, 92, 2121-2129.

M. Bernheim and F. Le Bourse, Nucl. Inst. Methods Phys. Res. B, 1987, 27, 94-103.

M. Bernheim and G. Slodzian, J. Phys. Lettres, 1977, 38, 325-328.

V. E. Krohn, J. Appl. Phys., 1962, 33, 3523-3525. H. A. Storms, K. F. Brown and J. D. Stein, Anal. Chem., 1977, 49, 2023-2030.

T. Wirtz and H. N. Migeon, Surf. Sci., 2004, 561, 200-207. K. Wittmaack, Surf. Sci. Rep., 2013, 68, 108-230. A. Priebe and J. Michler, Ultramicroscopy, 2019, 196, 1017.

J. A. Whitby, F. Östlund, P. Horvath, M. Gabureac, J. L. Riesterer, I. Utke, M. Hohl, L. Sedláček, J. Jiruše, V. Friedli, M. Bechelany and J. Michler, Adv. Mater. Sci. Eng., , DOI:10.1155/2012/180437. D. Alberts, L. von Werra, F. Oestlund, U. Rohner, M. Hohl, J. Michler and J. A. Whitby, Instrum. Sci. Technol., 2014, 42, 432-445.

L. Pillatsch, F. Östlund and J. Michler, Prog. Cryst. Growth Charact. Mater., 2019, 65, 1-19.

A. Priebe, T. Xie, G. Bürki, L. Pethö and J. Michler, J. Anal. At. Spectrom., 2020, 35, 1156-1166.

K. S. Sree Harsha, Principles of Vapor Deposition of Thin Films, Elsevier Science, Oxford, UK, 2006. M. Toth, C. Lobo, V. Friedli, A. Szkudlarek and I. Utke, Beilstein J. Nanotechnol., 2015, 6, 1518-1540. V. Friedli, H. D. Wanzenböck and I. Utke, in Nanofabrication using focused ion and electron beams: 
principles and applications, eds. I. Utke, S. Moshkalev and P. Russel, Oxford University Press, New York, 2012, pp. 126-183.

34 V. Friedli and I. Utke, J. Phys. D. Appl. Phys., , DOI:10.1088/0022-3727/42/12/125305.

35 B. Schmidt and K. Wetzig, Ion Beams in Materials Processing and Analysis, Springer-Verlag Wien, Viena, 2013.

36 W. G. Graham, Proc. Second Int. Symp. Prod. Neutralization Negat. Hydrog. lons Beams, 1980, 126.

37 J. Ishikawa, in The Physics and Technology of Ion Sources, ed. Ian G. Brown, Wiley-VCH Verlag GmbH \& Co. KGaA, 2nd edn., 2005, pp. 285-310.

38 R. L. Hervig, F. K. Mazdab, P. Williams, Y. Guan, G. R. Huss and L. A. Leshin, Chem. Geol., 2006, 227, 83-99.

39 L. Pillatsch, N. Vanhove, D. Dowsett, S. Sijbrandij, J. Notte and T. Wirtz, Appl. Surf. Sci., 2013, 282, 908-913.

40 L. Pillatsch and T. Wirtz, Surf. Interface Anal., 2012, 44, 1370-1372.

41 Z. M. Wang, FIB Nanostructures, Springer, Heidelberg, 2013.

42 A. Peter and J. de Paula, Atkins' Physical Chemistry, Oxford University Press, Oxford, 2014.

43 V. R. Deline, C. A. Evans and P. Williams, Appl. Phys. Lett., 1978, 33, 578-580.

44 K. Wittmaack, J. Appl. Phys., 1981, 52, 527-529.

45 P. Williams, V. R. Deline, C. A. Evans and W. Katz, J. Appl.

Phys., 1981, 52, 530-532. 